\title{
On the Loss of Wind-Induced Near-Inertial Energy to Turbulent Mixing in the Upper Ocean
}

\author{
Xiaoming Zhai,* Richard J. Greatbatch, and CARsten Eden \\ IFM-GEOMAR, Kiel, Germany \\ TOSHIYUKI HIBIYA \\ Department of Earth and Planetary Science, Graduate School of Science, University of Tokyo, Tokyo, Japan
}

(Manuscript received 27 March 2009, in final form 23 June 2009)

\begin{abstract}
Wind-induced near-inertial energy has been believed to be an important source for generating the ocean mixing required to maintain the global meridional overturning circulation. In the present study, the nearinertial energy budget in a realistic $1 / 12^{\circ}$ model of the North Atlantic Ocean driven by synoptically varying wind forcing is examined. The authors find that nearly $70 \%$ of the wind-induced near-inertial energy at the sea surface is lost to turbulent mixing within the top $200 \mathrm{~m}$ and, hence, is not available to generate diapycnal mixing at greater depth. Assuming this result can be extended to the global ocean, it is estimated that the wind-induced near-inertial energy available for ocean mixing at depth is, at most, $0.1 \mathrm{TW}$. This confirms a recent suggestion that the role of wind-induced near-inertial energy in sustaining the global overturning circulation might have been overemphasized.
\end{abstract}

\section{Introduction}

Near-inertial internal waves have been believed to be an important energy source for the diapycnal mixing in the ocean required to maintain the meridional overturning circulation (MOC) (Munk and Wunsch 1998). For example, the global annual-mean wind energy input to surface near-inertial motions has been estimated to be about 0.4 TW (e.g., Alford 2003a; Furuichi et al. 2008), a significant fraction of the $2 \mathrm{TW}$ energy thought to be necessary to support the upwelling branch of the MOC (Munk and Wunsch 1998). There are several redistributing processes that may be at work to transfer the near-inertial energy from the surface mixed layer downward. The traditional view is that near-inertial energy is redistributed in the ocean largely by the $\beta$-dispersion effect, whereby near-inertial internal waves are free to

* Current affiliation: University of Oxford, Oxford, United Kingdom.

Corresponding author address: Xiaoming Zhai, Atmospheric, Oceanic and Planetary Physics, Department of Physics, University of Oxford, Oxford OX1 3PU, United Kingdom.

E-mail: zhai@atm.ox.ac.uk propagate equatorward, and also downward, but are restricted in their poleward propagation by the planetary vorticity gradient (e.g., Anderson and Gill 1979; Gill 1984; Garrett 2001). Observational evidence has been found to support this idea (e.g., Chiswell 2003; Alford 2003b). On the other hand, it has been realized that the ocean is not homogeneous, and, similar to the idea of $\beta$ dispersion, the horizontal gradient of the relative vorticity can also influence the propagation of near-inertial internal waves (Kunze 1985; Young and Jelloul 1997; van Meurs 1998; Lee and Niiler 1998; Klein and Smith 2001; Zhai et al. 2005; Danioux et al. 2008). In particular, Mooers (1975) introduced the idea of the "effective Coriolis parameter" given by $f_{\text {eff }}=f+\zeta / 2$, where $f$ is the Coriolis parameter and $\zeta$ is the relative vorticity. Horizontal inhomogeneity of $f_{\text {eff }}$ can then lead to trapping of near-inertial energy inside regions of negative relative vorticity, for example, inside anticyclonic eddies [the "chimney effect" of Lee and Niiler (1998)], analogous to the trapping of near-inertial energy by the $\beta$-dispersion effect. Zhai et al. (2005) have pointed out that regions of strong near-inertial energy input from wind to the ocean are also regions of strong mesoscale variability in the ocean. This led Zhai et al. (2007) to carry out a numerical 
study on the interaction between near-inertial waves and mesoscale eddies using a realistic eddy-permitting $\left(1 / 12^{\circ}\right)$ model of the North Atlantic Ocean driven by synoptically varying wind forcing. They found that the wind-induced near-inertial energy at the sea surface can be drained locally into the thermocline by the mesoscale eddy field and, in particular, by the chimney effect associated with anticyclonic eddies, with no significant leaking equatorward through the $\beta$-dispersion effect. Evidence for enhanced near-inertial energy and associated turbulence and mixing has been found in anticyclonic eddies (e.g., Lueck and Osborn 1986; Kunze et al. 1995). However, a quantitative picture, that is, how much of the nearinertial energy input at the sea surface is actually available for mixing in the ocean interior, is still lacking. Recently, Furuichi et al. (2008) investigated the distribution of wind-induced internal wave energy in the world's oceans using a $1 / 7^{\circ}$ (in longitude) model forced by 6-hourly wind stress computed from the Japanese 25-year reanalysis (JRA-25) (Onogi et al. 2007). They inferred that $75 \%-85 \%$ of the near-inertial energy input at the sea surface is dissipated in the top $150 \mathrm{~m}$, leaving only a limited amount of energy $(<0.1 \mathrm{TW})$ available for mixing below 150-m depth. Their model, however, did not include the mesoscale eddy field or the mean flow so that spatial inhomogeneity due to relative vorticity in the effective Coriolis parameter, $f_{\text {eff }}$, was not considered.

In the present study, we examine the near-inertial energy budget in the upper ocean in a realistic $1 / 12^{\circ}$ model of the North Atlantic Ocean, driven by synoptically varying wind forcing, that includes both the $\beta$-dispersion and eddy chimney effects. In particular, we directly calculate the viscous removal of near-inertial energy and quantify the amount of near-inertial energy escaping from the upper ocean that may potentially be available for ocean mixing at depth.

\section{The near-inertial energy budget}

We separate the variables into their subinertial, inertial, and turbulent components. The governing equation for the near-inertial energy can be derived from the momentum and continuity equations under the hydrostatic and Boussinesq approximations such that

$$
\begin{aligned}
\frac{\partial E_{i}}{\partial t}= & -\nabla \cdot\left(\mathbf{U} E_{i}\right)-\nabla \cdot\left(p_{i} \mathbf{u}_{i}\right)+\frac{\partial}{\partial z}\left(K_{v} \frac{\partial E_{i}}{\partial z}\right)-\rho_{i} w_{i} g \\
& -\rho_{0} K_{v}\left|\frac{\partial \mathbf{u}_{i}}{\partial z}\right|^{2}+\text { others }
\end{aligned}
$$

where $\mathbf{U}$ is the background subinertial velocity and subscript $i$ denotes near-inertial filtered quantities, $E_{i}=$ $\rho_{0}\left(u_{i}^{2}+v_{i}^{2}\right) / 2$ is the near-inertial kinetic energy per unit volume, $K_{v}$ is the vertical turbulent viscosity coefficient, and $\boldsymbol{\nabla}$ is the three-dimensional gradient operator. The terms on the rhs of Eq. (1) are the advection of nearinertial kinetic energy by the background subinertial flow (e.g., Zhai et al. 2004), the divergence of nearinertial energy flux, the vertical diffusion of near-inertial kinetic energy with the boundary condition $K_{v}\left(\partial E_{i} / \partial z\right)=$ $\boldsymbol{\tau} \cdot \mathbf{u}_{i}$ at the sea surface where $\boldsymbol{\tau}$ is the surface wind stress, the conversion between near-inertial kinetic energy and near-inertial potential energy, and the near-inertial kinetic energy sink caused by vertical viscous effects that corresponds to the shear production term in the turbulent kinetic energy (TKE) equation. Processes associated with subgrid-scale horizontal viscosity and nonlinear energy transfers from/to other frequencies are denoted by "others" in Eq. (1). In the model, horizontal mixing is parameterized using a biharmonic viscosity (see section 3).

For stationary and horizontally homogeneous turbulence, the TKE balance can be expressed as

$$
-\rho_{0} \overline{\mathbf{u}^{\prime} w^{\prime}} \cdot \frac{\partial \mathbf{u}_{i}}{\partial z}-\overline{\rho^{\prime} w^{\prime}} g-\varepsilon=0,
$$

where primed variables denote turbulent quantities, and the terms on the lhs are the shear production of TKE, the buoyancy production of TKE, and the viscous dissipation of TKE, respectively. In the vertical mixing scheme used in our model (Gaspar et al. 1990), the vertical turbulent momentum flux is parameterized in terms of the classical concept of eddy diffusivity, namely

$$
-\overline{\mathbf{u}^{\prime} w^{\prime}}=K_{v} \frac{\partial \mathbf{u}_{i}}{\partial z} .
$$

Then, (2) becomes

$$
\rho_{0} K_{v}\left|\frac{\partial \mathbf{u}_{i}}{\partial z}\right|^{2}=\overline{\rho^{\prime} w^{\prime}} g+\varepsilon .
$$

Consistent with mixing efficiencies estimated in laboratory experiments and observations (e.g., Ruddick et al. 1997), we might assume that about $25 \%$ of the viscous removal of near-inertial energy by this vertical mixing scheme is used to increase the potential energy of the water column (e.g., through entrainment and mixed layer deepening via the buoyancy production term), with the rest simply dissipated into heat.

Integrating (1) over a fixed volume of interest and ignoring the advective and diffusive transports of nearinertial kinetic energy across the open boundaries (which, in our case, are shown to be smaller by comparison), we can get the near-inertial kinetic energy budget for the upper ocean: 


$$
\begin{aligned}
\frac{\partial}{\partial t} \int E_{i} d V= & -\int\left(p_{i} \mathbf{u}_{i}\right) d A_{I}+\int\left(\boldsymbol{\tau} \cdot \mathbf{u}_{i}\right) d A_{S} \\
& -\int\left(\rho_{i} w_{i} g\right) d V-\int\left(\rho_{0} K_{v}\left|\frac{\partial \mathbf{u}_{i}}{\partial z}\right|^{2}\right) d V \\
& +\int(\text { others }) d V,
\end{aligned}
$$

where $V$ is the control volume, $A_{S}$ is the sea surface area, $A_{I}$ is the area of the side and bottom open boundaries of the control volume, and $\boldsymbol{\tau} \cdot \mathbf{u}_{i}$ is the near-inertial energy input by wind at the sea surface. In physical terms, Eq. (5) states that the change of near-inertial kinetic energy within this control volume is caused by input of wind-induced near-inertial energy at the sea surface, the removal of near-inertial energy caused by subgrid-scale vertical viscosity, the conversion from near-inertial kinetic energy to near-inertial potential energy within the control volume, and the near-inertial energy flux through the open boundaries, plus the removal of near-inertial energy caused by subgrid-scale horizontal viscosity as well as nonlinear energy transfers to other frequency bands. In the present study, we diagnose each term in (5) using the numerical model described in the next section.

\section{The model}

The model used in this study is based on a revised version of MOM2, and is identical to the one used by Zhai et al. (2007). The horizontal resolution is $\sim 10 \mathrm{~km}$ at the equator decreasing to $\sim 5 \mathrm{~km}$ at high latitudes, corresponding to roughly $1 / 12^{\circ}$ in longitude. The model domain extends between the open boundaries at $20^{\circ} \mathrm{S}$ and $70^{\circ} \mathrm{N}$, formulated following Stevens (1990), with a restoring zone in the eastern Mediterranean Sea. There are 45 vertical geopotential levels with increasing thickness with depth, ranging from $10 \mathrm{~m}$ at the sea surface to $250 \mathrm{~m}$ near the maximal depth of $5500 \mathrm{~m}$. The model adopts the vertical mixing scheme of Gaspar et al. (1990), which includes a single prognostic equation for TKE, and diagnoses the mixing length scales defined as the distances traveled upward or downward by a fluid parcel in converting its TKE to potential energy. Biharmonic friction is used in the model with a viscosity coefficient of $2 \times 10^{10} \cos (\phi) \mathrm{m}^{4} \mathrm{~s}^{-1}$ ( $\phi$ is the latitude). The model was first spun up for 10 years under monthly climatological forcing. After that, it was forced using daily wind stress data taken from the ECMWF operational weather forecast model available at 1200 UTC on each day from year 2001 to 2004. The horizontal resolution of the ECMWF data is about $40 \mathrm{~km} \times 40 \mathrm{~km}$, much higher than the $2.5^{\circ}$ resolution of the wind field from the National Centers for Environmental Prediction-National
Center for Atmospheric Research (NCEP-NCAR) reanalysis product. We use here forcing starting on 1 January 2001. Note that, even though our wind forcing has relatively high spatial resolution, the use of daily wind stress is likely to underestimate the wind near-inertial energy input to the ocean (e.g., Niwa and Hibiya 1999; Davies and Xing 2004; Furuichi et al. 2008). Nevertheless, the overall pattern and magnitude of near-inertial energy input in our model is consistent with those obtained in the previous studies (see section 4a).

The model variables $\left(u, v, \rho, K_{v}, \tau\right)$ are saved every 0.1 days. The baroclinic horizontal velocities and perturbation pressure fields are obtained by subtracting their depth averages, and the vertical velocity is computed from the continuity equation using baroclinic horizontal velocities. Unless otherwise stated, we are concerned only with the baroclinic component in our energy budget analysis since the barotropic near-inertial energy is negligible. To compute near-inertial energy, the horizontal velocity is filtered (using a Butterworth filter) to retain periods between 0.8 and 1.5 days, which correspond to the inertial periods at $23^{\circ}$ and $45^{\circ} \mathrm{N}$, respectively. These cutoff periods are sufficient for the region of our interest (see Fig. 1), and further refinement of the bandpass filter does not lead to any noticeable changes of the near-inertial properties in the model. Note also that the contamination of near-inertial energy from the movement of eddies at near-inertial frequency is negligible (e.g., Zhai et al. 2005; Danioux et al. 2008). Our energy budget analysis is limited to a region extending from $25^{\circ}$ to $42^{\circ} \mathrm{N}, 75^{\circ}$ to $30^{\circ} \mathrm{W}$ (Fig. 1a). This region is of interest since it covers areas of strong nearinertial energy input at the sea surface and also includes a rigorous mesoscale eddy field. The practical reason for choosing such a limited region is that the dataset for the whole model domain is too large to analyze.

\section{Results}

\section{a. Near-inertial energy source and sink}

The offline calculation of terms in (5) follows the model algorithm, which has a B-grid configuration. The contribution of each term to the near-inertial energy budget in our selected region is summarized in Table 1 . Figure $1 b$ shows the wind input of near-inertial energy at the sea surface calculated using $\boldsymbol{\tau} \cdot \mathbf{u}_{i}$ and integrated for 13 days starting from 3 March. The overall pattern and magnitude are very similar to those in Zhai et al. (2007) and also broadly consistent with the estimates given by Alford (2003a) and Furuichi et al. (2008). The nearinertial energy input is largely positive and concentrated in the northern half of the region, which corresponds to the atmospheric storm track. The spatially and temporally 

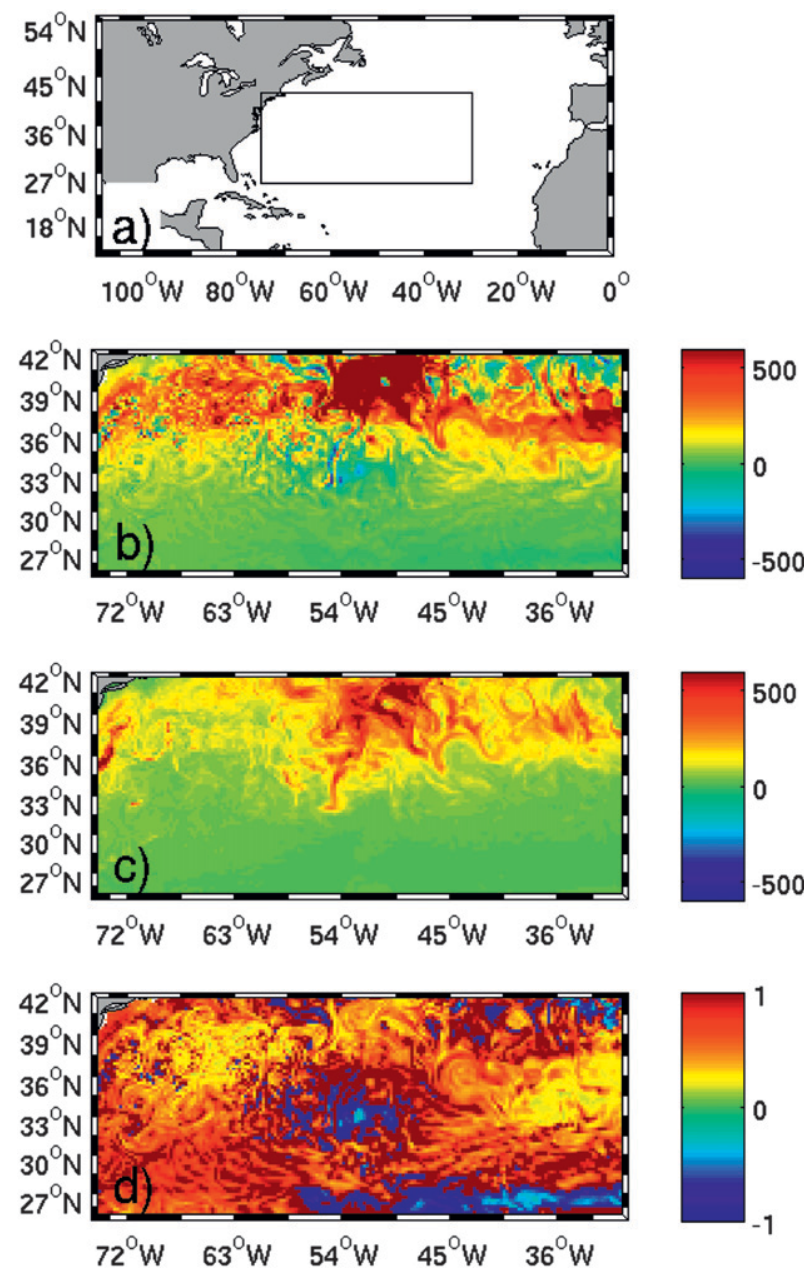

FIG. 1. (a) The rectangular box shows the region where the energy budget analysis is conducted; (b) near-inertial energy input $\left(\mathrm{N} \mathrm{m}^{-1}\right)$ by wind at the sea surface; (c) near-inertial energy lost within the top $234 \mathrm{~m}$ due to vertical viscous effects in $\mathrm{N} \mathrm{m}^{-1}$; and (d) the ratio of (c) to (b).

integrated near-inertial energy input is $8.7 \times 10^{14} \mathrm{~N} \mathrm{~m}$. The complex pattern reflects the surface mesoscale eddy field, even though mesoscale eddies may not significantly affect the area-integrated total near-inertial energy input (e.g., Klein et al. 2004). Not surprisingly, viscous removal of near-inertial energy mostly takes place where high values of near-inertial energy are found and, therefore, decreases with depth. The total sink of near-inertial energy amounts to $5.9 \times 10^{14} \mathrm{~N} \mathrm{~m}$ when integrated over the top $234 \mathrm{~m}$ and for the same 13-day period (Fig. 1c). Figure 1d shows the ratio of Fig. 1c to 1b. Over most of the region, the near-inertial energy lost due to viscous effects is a significant fraction of the surface energy input by wind. Integrated over the volume, $68 \%$ of the total wind-induced near-inertial energy is lost within the top $234 \mathrm{~m}$ in the ocean. Our direct estimate of the near-inertial energy sink in the upper ocean is a bit lower than inferred by Furuichi et al. (2008) $(75 \%-85 \%)$. However, the dissipation of nearinertial energy associated with the lateral biharmonic friction employed in the model amounts to $8 \%$ of the wind-induced near-inertial energy, sufficient to explain the difference. Our model estimate thus provides direct evidence that a significant fraction of wind-driven nearinertial energy is lost to turbulent mixing in the upper ocean, without contributing to diapycnal mixing at greater depth. This is the most important result of the present study, supporting the recent claim by Furuichi et al. (2008). Consistent with mixing efficiencies estimated in laboratory experiments and observations, about $25 \%$ of the near-inertial energy viscously removed from the energy budget is used in the model to increase the potential energy of the upper ocean, for example, for mixed layer entrainment and deepening.

\section{b. Near-inertial energy flux and conversion}

Figure 2a shows the vertical near-inertial energy flux $\left(p_{i} w_{i}\right)$ across the bottom open boundary at a depth of $234 \mathrm{~m}$ integrated for the 13-day period. It shows alternating wave patterns in the interior of the subtropical gyre, that is, to the south of $35^{\circ} \mathrm{N}$, but becomes more negative in the Gulf Stream extension region, suggesting the crucial role of eddies in draining the near-inertial energy downward. The total downward near-inertial energy flux in Fig. 2a sums up to $8.7 \times 10^{13} \mathrm{~N} \mathrm{~m}$, that is, $10 \%$ of the total near-inertial energy input at the sea surface. Careful analysis reveals that more than $90 \%$ of this downward energy flux occurs in the region with vigorous mesoscale eddies. The ratio of the downward near-inertial energy flux at a depth of $234 \mathrm{~m}$ to the surface near-inertial energy input is almost twice that in Furuichi et al. (2008). This is presumably because mesoscale eddies are taken into account in our $1 / 12^{\circ}$ model, but not in theirs. The mesoscale eddies, especially the anticyclonic eddies, can efficiently drain the near-inertial energy to great depths (e.g., Zhai et al. 2007). The conversion from near-inertial kinetic energy to potential energy is at least two orders of magnitude smaller compared to the other terms (not shown).

Figures $2 \mathrm{~b}$ and $2 \mathrm{c}$ show the horizontal near-inertial energy flux averaged in time and over the top $234 \mathrm{~m}$. We do observe equatorward energy propagation, especially between $60^{\circ}$ and $45^{\circ} \mathrm{W}$ where the maximum near-inertial energy input is found north of $36^{\circ} \mathrm{N}$. However, when integrated along the southern open boundary, it only accounts for $1 \%$ of the total input of the near-inertial energy by wind over the region. The zonal near-inertial energy flux has similar magnitude and shows divergence in the region of the maximum near-inertial energy input. The 
TABLE 1. The near-inertial energy budget for the top $234 \mathrm{~m}$ of the region bounded by the box shown in Fig. 1a and integrated for the 13-day period: $A_{I b}$ is the area of the bottom open boundary, $A_{I S}$ is that of the southern boundary, and $A_{I o}$ is that of the other lateral boundaries of the volume. The number in parentheses is the ratio of each energy term to the wind near-inertial energy input.

\begin{tabular}{llc}
\hline \hline \multicolumn{1}{c}{ Energy term in (5) } & & Contribution (ratio to wind energy input) \\
\hline Wind near-inertial energy input & $\int\left(\boldsymbol{\tau} \cdot \mathbf{u}_{i}\right) d A_{S}$ & $8.7 \times 10^{14} \mathrm{~N} \mathrm{~m}$ \\
Viscous removal in the top $234 \mathrm{~m}$ & $\int\left(\rho_{0} K_{v}\left|\partial \mathbf{u}_{i} / \partial z\right|^{2}\right)$ & $5.9 \times 10^{14} \mathrm{~N} \mathrm{~m}(68 \%)$ \\
Vertical near-inertial energy flux & $\int\left(p_{i} w_{i}\right) d A_{I b}$ & $8.7 \times 10^{13} \mathrm{~N} \mathrm{~m}(10 \%)$ \\
Southward energy flux through $A_{I S}$ & $\int\left(p_{i} v_{i}\right) d A_{I s}$ & $7.0 \times 10^{12} \mathrm{~N} \mathrm{~m}(<1 \%)$ \\
Energy flux through $A_{I o}$ & $\int\left(p_{i} \mathbf{u}_{i}\right) d A_{I o}$ & $2.0 \times 10^{13} \mathrm{~N} \mathrm{~m}(2 \%)$ \\
Conversion to inertial potential energy & $\int\left(\rho_{i} w_{i} g\right) d V$ & $\mathrm{Negligible}$ \\
Others: biharmonic dissipation & $(8 \%)$ \\
The remainder & & $(\sim 12 \%)$ \\
\hline
\end{tabular}

effect of advection through the open boundaries is indeed small and can be neglected in our energy budget analysis.

\section{Discussion and summary}

Wind-driven near-inertial internal waves have been believed to be an important energy source for the diapycnal mixing in the ocean interior required to maintain the global meridional overturning circulation. Both the $\beta$ dispersion (e.g., Anderson and Gill 1979; Gill 1984; Garrett 2001) and eddy chimney effects (e.g., Zhai et al. 2005; Zhai et al. 2007) can transfer the wind-induced near-inertial energy from the surface mixed layer into the ocean interior. However, a quantitative assessment of the wind-induced near-inertial energy available for mixing in the ocean interior has been lacking. In the present study, we have addressed this issue by examining the near-inertial energy budget in the upper ocean in a realistic $1 / 12^{\circ}$ model of the North Atlantic Ocean driven by synoptically varying wind forcing. Both $\beta$ dispersion and eddy chimney effects have been included in our model. The present study has shown that almost $70 \%$ of the near-inertial energy input by wind at the sea surface is lost to vertical turbulent mixing within the top $200 \mathrm{~m}$, such that the total near-inertial energy that may potentially be available for mixing at depth is only a small fraction of the energy input at the surface. This is consistent with a recent study by Furuichi et al. (2008), who inferred significant dissipation $(75 \%-85 \%)$ of the windinduced near-inertial energy in the surface $150 \mathrm{~m}$ using a model with no eddies. Although longer integrations using wind stress forcing with higher temporal and spatial resolutions are required, our model results have clearly demonstrated that most of the wind-induced near-inertial energy in our model is lost to increase potential energy and internal energy in the upper $200 \mathrm{~m}$ of the ocean, and hence is not available to generate diapycnal mixing at greater depth.

The annual mean global wind near-inertial energy input to the ocean at the sea surface has been estimated to be $\sim 0.4$ TW (e.g., Watanabe and Hibiya 2002; Alford 2003a; Furuichi et al. 2008). Assuming that our result can be extended to the global ocean, we estimate that the

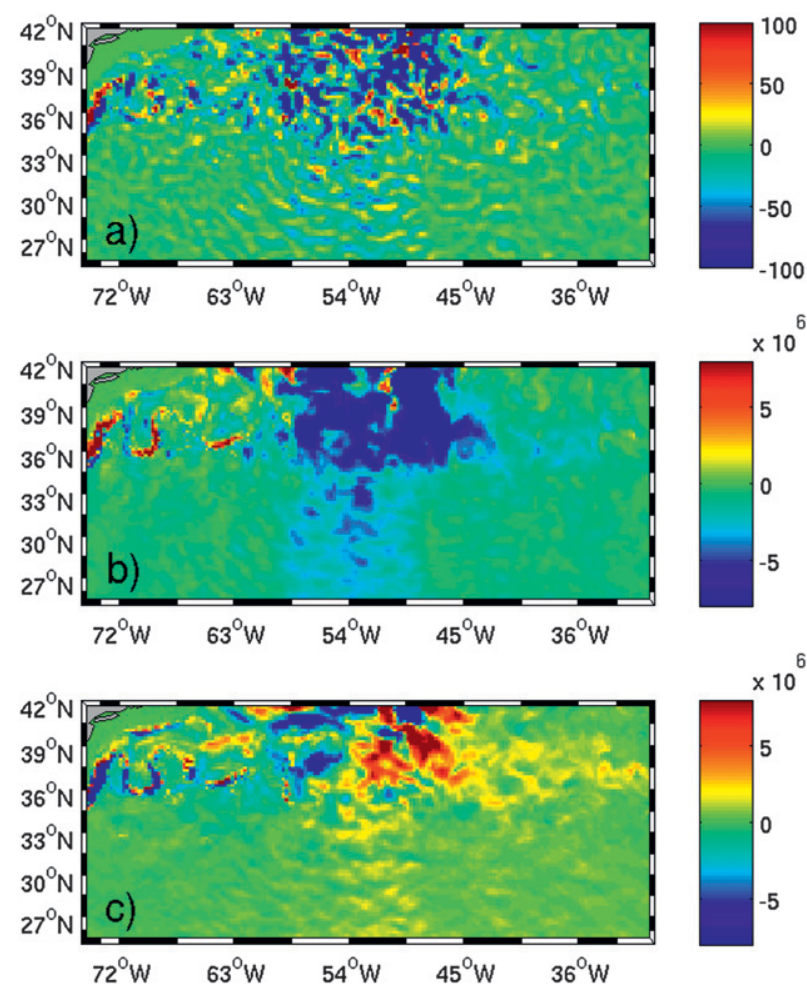

FIG. 2. (a) Vertical near-inertial energy flux, $p_{i} w_{i},\left(\mathrm{~N} \mathrm{~m}^{-1}\right)$ at a depth of $234 \mathrm{~m}$ integrated for the 13-day period; (b) $p_{i} v_{i}$ and (c) $p_{i} u_{i}$ integrated over the top $234 \mathrm{~m}$ for the 13-day period. 
wind-induced near-inertial energy available for ocean mixing at depth is, at most, $0.1 \mathrm{TW}$. This confirms a recent suggestion by Furuichi et al. (2008) that the role of the wind-induced near-inertial energy in sustaining the global overturning circulation might have been overemphasized. Other sources (e.g., geostrophic adjustment processes, tides) for generating near-inertial waves and diapycnal mixing in the ocean interior need to be considered.

Acknowledgments. XZ wishes to thank Lars Czeschel for help with the model. This work has been funded by IFM-GEOMAR. We are grateful to two anonymous reviewers for helpful comments on the manuscript.

\section{REFERENCES}

Alford, M. H., 2003a: Improved global maps and 54-years history of wind-work on ocean inertial motions. Geophys. Res. Lett., 30, 1424, doi:10.1029/2002GL016614.

__ 2003b: Redistribution of energy available for ocean mixing by long-range propagation of internal waves. Nature, 423, 159-162.

Anderson, D. L. T., and A. E. Gill, 1979: Beta dispersion of inertial waves. J. Geophys. Res., 84, 1836-1842.

Chiswell, S. M., 2003: Deep equatorward propagation of inertial oscillations. Geophys. Res. Lett., 30, 1533, doi:10.1029/ 2003 GL017057.

Danioux, E., P. Klein, and P. Riviére, 2008: Propagation of wind energy into the deep ocean through a fully turbulent mesoscale eddy field. J. Phys. Oceanogr., 38, 2224-2241.

Davies, A. M., and J. Xing, 2004: Wind induced motion in the vicinity of a bottom density front: Response to forcing frequency. J. Geophys. Res., 109, C09002, doi:10.1029/2003JC002151.

Furuichi, N., T. Hibiya, and Y. Niwa, 2008: Model-predicted distribution of wind-induced internal wave energy in the world's oceans. J. Geophys. Res., 113, C09034, doi:10.1029/2008JC004768.

Garrett, C., 2001: What is the "near-inertial" band and why is it different from the rest of the internal wave spectrum $J$. Phys. Oceanogr., 31, 962-971.

Gaspar, P., Y. Grégoris, and J. M. Lefevre, 1990: A simple eddy kinetic energy model for simulations of the oceanic vertical mixing: Tests at station papa and long-term upper ocean study site. J. Geophys. Res., 95, 16 179-16 193.

Gill, A. E., 1984: On the behavior of internal waves in the wakes of storms. J. Phys. Oceanogr., 14, 1129-1151.

Klein, P., and S. G. L. Smith, 2001: Horizontal dispersion of nearinertial oscillations in a turbulent mesoscale eddy field. J. Mar. Res., 59, 697-723.
— G. Lapeyre, and W. G. Large, 2004: Wind ringing of the ocean in presence of mesoscale eddies. Geophys. Res. Lett., 31, L15306, doi:10.1029/2004GL020274.

Kunze, E., 1985: Near-inertial propagation in geostrophic shear. J. Phys. Oceanogr., 15, 544-565.

— , R. W. Schwitt, and J. M. Toole, 1995: The energy balance in a warm-core ring's near-inertial critical layer. J. Phys. Oceanogr., 25, 942-957.

Lee, D. K., and P. P. Niiler, 1998: The inertial chimney: The nearinertial energy drainage from the ocean surface to the deep layer. J. Geophys. Res., 103, 7579-7591.

Lueck, R., and T. Osborn, 1986: The dissipation of kinetic energy in a warm-core ring. J. Geophys. Res., 91, 803-818.

Mooers, C. N. K., 1975: Several effects of a baroclinic current on the cross-stream propagation of inertial-internal waves. Geophys. Fluid Dyn., 6, 245-275.

Munk, W., and C. Wunsch, 1998: Abyssal recipes II, Energetics of tidal and wind mixing. Deep Sea Res. I, 45, 1977-2010.

Niwa, Y., and T. Hibiya, 1999: Response of the deep ocean internal wave field to traveling midlatitude storms as observed in longterm current measurements. J. Geophys. Res., 104, 10 98110989.

Onogi, K., and Coauthors, 2007: The JRA-25 reanalysis. J. Meteor. Soc. Japan, 85, 369-432.

Ruddick, B., D. Walsh, and N. Oakey, 1997: Variations in apparent mixing efficiency in the North Atlantic Center Water. J. Phys. Oceanogr., 27, 2589-2605.

Stevens, D. P., 1990: On open boundary conditions for three dimensional primitive equation ocean circulation models. Geophys. Astrophys. Fluid Dyn., 51, 103-133.

van Meurs, P., 1998: Interactions between near-inertial mixed layer currents and the mesoscale: The importance of spatial variabilities in the vorticity field. J. Phys. Oceanogr., 28, 13631388.

Watanabe, M., and T. Hibiya, 2002: Global estimates of the windinduced energy flux to inertial motions in the surface mixed layer. Geophys. Res. Lett., 29, 1239, doi:10.1029/2001GL014422.

Young, W. R., and M. B. Jelloul, 1997: Propagation of near-inertial oscillations through a geostrophic flow. J. Mar. Res., 55, 735-766.

Zhai, X., R. J. Greatbatch, and J. Sheng, 2004: Advective spreading of storm-induced inertial oscillations in a model of the northwest Atlantic Ocean. Geophys. Res. Lett., 31, L14315, doi:10.1029/2004GL020084.

—_ - and J. Zhao, 2005: Enhanced vertical propagation of storm-induced near-inertial energy in an eddying ocean channel model. Geophys. Res. Lett., 32, L18602, doi:10.1029/ 2005 GL023643.

,-- , and C. Eden, 2007: Spreading of near-inertial energy in a $1 / 2^{\circ}$ model of the north Atlantic Ocean. Geophys. Res. Lett., 34, L10609, doi:10.1029/2007GL029895. 